\title{
Risco de fadiga prematura, percepção subjetiva de esforço e estratégia de prova durante uma corrida de $10 \mathrm{~km}$
}

CDD. 20.ed. 796.073

796.426

http://dx.doi.org/10.1590/1807-55092015000200197

\author{
Everton Crivoi do CARMO**** \\ Saulo GIL* \\ Salomão BUENO* \\ Leonardo Alves PASQUE* \\ Adriano Eduardo LIMA-SILVA*** \\ Rômulo BERTUZZI* \\ Valmor TRICOLI ${ }^{*}$ \\ *Escola de Educa- \\ ção Física e Esporte, \\ Universidade de São \\ Paulo. \\ ${ }^{* *}$ Centro Universitário \\ SENAC \\ ${ }^{* * *}$ Centro de Ciências \\ da Saúde, Universi- \\ dade Federal de Per- \\ nambuco.
}

\section{Resumo}

0 objetivo do estudo foi verificar as modificações na estratégia de prova frente às alterações do risco de fadiga prematura e da percepção subjetiva de esforço (PSE) em corredores durante uma corrida de $10 \mathrm{~km}$. Participaram do estudo 55 corredores com tempo nos $10 \mathrm{~km}$ de 41:39 \pm 3:52 min:s. A estratégia de prova e a PSE foram avaliadas a cada quilômetro. 0 risco de fadiga prematura foi determinado pelo produto entre a PSE e a distância restante de prova e a estratégia de prova foi determinada pela curva da velocidade e distância. A ANOVA de um caminho para medidas repetidas foi utilizada para determinar as diferenças na velocidade, PSE e risco de fadiga a cada quilômetro e entre a velocidade a cada quilômetro e a velocidade média da prova. 0 coeficiente de correlação de Pearson foi calculado entre a PSE e o risco de fadiga prematura com a velocidade. A velocidade do primeiro quilômetro foi $8,1 \%$ maior do que a média $(\mathrm{p} \leq$ $0,001)$. A velocidade diminuiu gradualmente ao longo da prova, ocorrendo um novo aumento no décimo quilômetro. A PSE aumentou linearmente ao longo da prova e o risco de fadiga diminuiu significantemente após o terceiro quilômetro. Houve forte correlação negativa entre a PSE e a velocidade desenvolvida durante a prova $(r=-0,80 ; p=0,006)$. Foi observada uma correlação moderada negativa entre o risco de fadiga prematura e a velocidade $(r=-0,57 ; p=0,04)$. Com isso, os achados do presente estudo sugerem que a PSE parece ter importante papel sobre os ajustes da velocidade ao longo da prova, sendo que o aumento da velocidade observado no último quilômetro pode estar associado ao baixo risco de fadiga prematura.

Palavras-chave: Desempenho atlético; Consumo energético; Tolerância ao exercício; Fadiga.

\section{Introdução}

Os ajustes constantes de velocidade durante uma corrida são denominados estratégia de prova $^{1-3}$ e têm sido considerados fatores determinantes para o sucesso de atletas em provas de média e longa distâncias ${ }^{2,4}$. Dentre as diferentes estratégias observadas ${ }^{2,5}$, acreditase que a mais eficaz é aquela em que o atleta é capaz de regular seu gasto energético para prevenir a fadiga prematura e realizar a prova no menor tempo possível ${ }^{6-7}$. Para isso, um controle eficiente da intensidade do esforço e da estratégia ao longo da prova é necessário.

Tem sido proposto que o controle da estratégia de prova é realizado a partir de alteraçóes em fatores fisiológicos e psicológicos, as quais são ajustadas momento a momento durante a prova ${ }^{8-11}$. Estas alteraçóes induzidas pelo exercício podem ser expressas conscientemente pela escala de percepção subjetiva de esforço (PSE). De fato, a PSE tem sido apontada como uma importante ferramenta no controle da estratégia de prova ${ }^{12-15}$. Durante uma corrida os atletas comparariam a PSE esperada para aquele determinado momento, baseada nas experiências prévias e na distância restante de prova, com a PSE momentânea, gerada pelas alteraçóes fisiológicas e psicológicas induzidas pelo exercício ${ }^{16}$. Se a PSE 
momentânea estiver menor do que a esperada entáo a velocidade é aumentada, caso contrário, se a PSE momentânea estiver maior que a esperada, entáo a velocidade é reduzida, a fim de atingir os maiores valores apenas ao final da prova, evitando assim uma possível fadiga prematura e queda de desempenho ${ }^{16}$.

Uma vez que a estratégia de prova parece ser ajustada para evitar uma possível fadiga prematura, DE Koning et al. ${ }^{17}$ propuseram um escore para determinação do risco de fadiga durante a corrida. Para a construção desse escore, os autores utilizaram o produto da PSE momentânea (utilizando a escala CR10), pela distância restante de prova. Assim, durante a corrida o atleta ajustaria sua velocidade constantemente a fim de manter uma PSE aceitável para a distância restante de prova, controlando o risco de fadiga prematura. De fato, os autores mostraram que os ajustes da velocidade durante uma competição parecem ser determinados pelo risco de fadiga, ou seja, pela relação entre a PSE e a distância restante de prova.

No entanto, os experimentos de De Koning et al. ${ }^{17}$ foram realizados em ciclo-simuladores ou em esteiras, onde os sujeitos foram forçados a correr os primeiros $4 \mathrm{~km}$ em uma velocidade pré-determinada $\mathrm{e}$ apenas após isso foram livres para ajustar a velocidade.

\section{Método}

\section{Amostra}

Participaram do estudo 55 corredores amadores, todos do sexo masculino, com idade entre 21 e 46 anos (TABELA 1). Como critério de inclusão no estudo os sujeitos deveriam treinar pelo menos quatro vezes por semana, com volume semanal acima de $40 \mathrm{~km}$, e possuir mais de três anos de experiência em corridas de média e longa duração. Os corredores não possuíam nenhum tipo de lesão ou patologia que pudesse oferecer riscos aos indivíduos ou influenciar os resultados do estudo. Os procedimentos adotados no estudo foram aprovados pelo Comitê de Ética e Pesquisa local. Todos os sujeitos receberam esclarecimentos sobre os procedimentos experimentais e os possíveis riscos e benefícios envolvidos e assinaram um termo de consentimento livre e esclarecido antes da participação no estudo.

\section{Procedimentos experimentais}

Todos os sujeitos foram submetidos a duas sessôes de familiarização com a escala de percepção
Adicionalmente, os ajustes de velocidade realizados na esteira podem ser substancialmente diferentes dos realizados em uma situação em pista, onde o atleta pode variar a sua velocidade livremente. Assim, as influências da PSE e do risco de fadiga prematura sobre os ajustes de velocidade realizados durante uma corrida de $10 \mathrm{~km}$ realizada em pista ainda são desconhecidos.

O papel da PSE sobre a estratégia de prova tem sido demonstrado na literatura, sendo que os atletas parecem ajustar a intensidade do esforço para atingir os máximos valores de PSE próximo ao final da corrida $^{16,18-20}$. Entretanto, o papel do risco de fadiga prematura sobre os ajustes da estratégia de prova tem sido pouco estudado.

Com isso, o objetivo do presente estudo foi verificar as modificaçóes induzidas sobre a estratégia de prova frente às alteraçóes do risco de fadiga prematura e da PSE em corredores amadores durante uma corrida simulada de $10 \mathrm{~km}$. A hipótese sugerida foi que a estratégia de prova utilizada pelos atletas durante uma corrida em pista poderia ser influenciada pela PSE e pelo risco de fadiga prematura. $\mathrm{O}$ melhor entendimento dos fatores que influenciam os ajustes da velocidade ao longo de uma prova poderá auxiliar atletas e treinadores no desenvolvimento de novas estratégias de corrida.

de esforço e para o reconhecimento dos locais dos testes. Após as familiarizaçôes, os sujeitos foram submetidos a duas sessóes experimentais separadas por no mínimo 72 horas. Na primeira sessão foi realizado o teste progressivo máximo para medição do consumo máximo de oxigênio $\left(\mathrm{VO}_{2}\right.$ máx). Durante a segunda sessáo foi realizada uma corrida simulada de $10 \mathrm{~km}$ em pista de $400 \mathrm{~m}$, para avaliação da estratégia de prova utilizada, onde foram mensuradas a velocidade e a PSE a cada volta.

\section{Teste progressivo máximo}

O teste foi iniciado com três minutos de aquecimento composto de corrida em esteira à velocidade de $8 \mathrm{~km} \cdot \mathrm{h}^{-1}$. Em seguida, foram realizados incrementos de $1 \mathrm{~km} \cdot \mathrm{h}^{-1}$ a cada minuto, até que o sujeito fosse incapaz de se exercitar, o que foi indicado pelo participante. O teste foi conduzido em esteira ergométrica (Super ATL, Inbrasport, Porto Alegre, Brasil) e as análises dos gases expirados foram realizadas respiração a respiração por um analisador de gases 
(Quark, Cosmed, Roma, Itália). O equipamento foi calibrado previamente conforme as especificaçóes contidas no manual do fabricante e os valores respiração a respiração do consumo de oxigênio foram convertidos em médias de 30 segundos $\left(\mathrm{VO}_{2}\right)$. A frequência cardíaca máxima (FCM) foi mensurada por meio de cardiofrequencímetro (Polar ${ }^{\oplus}$, modelo RS800CX, Finlândia) e definida como o maior valor obtido ao final do teste. $\mathrm{O} \mathrm{VO}_{2}$ máx foi considerado quando identificado platô do $\mathrm{VO}_{2}$ apesar do aumento da velocidade ou quando pelo menos dois dos seguintes critérios foram atingidos: 1) coeficiente respiratório maior que $1(\mathrm{RER}>1) ; 2)$ frequência cardíaca máxima atingida maior que $90 \%$ da predita pela idade (220-idade); e 3) percepção subjetiva de esforço exaustiva (classificação entre 19-20 na escala de 15 pontos de Borg) ${ }^{21}$.

\section{Corrida simulada de $10 \mathrm{~km}$}

\section{Estratégia de prova}

Os sujeitos realizaram uma corrida simulada de $10 \mathrm{~km}$ em uma pista de atletismo de $400 \mathrm{~m}$, sem a presença de adversários ou outro competidor na pista no mesmo momento do teste. Todos foram instruídos para completarem a prova o mais rápido possível. Durante a corrida, foi disponibilizada água "ad libitum".

A PSE foi coletada a cada 400 metros, sendo a escala de 15 pontos de Borg ${ }^{22}$ fixada ao antebraço dos participantes. Para a melhor padronizaçáo na orientação aos sujeitos sobre a utilização da escala, foram adotados os procedimentos sugeridos por HARDY e REJESKI ${ }^{23}$, sendo utilizada a seguinte explicação:

Ao participar de um exercício é muito comum sentirmos o quão intenso nós estamos trabalhando. Para responder a escala, gostaríamos que você considerasse a quantidade total de esforço que você sente, tendo em conta todas as sensaçôes de estresse físico, esforço e fadiga em todo o seu corpo. Os cientistas desenvolveram uma escala para medir essa percepção de esforço.

Nesse momento, a escala foi apresentada e explicada aos sujeitos.

A velocidade e a frequência cardíaca foram mensuradas com o uso do Sistema de Posicionamento Global (GPS Polar ${ }^{\bullet}$, modelo RS800CX, Finlândia) o qual permitiu a medição da velocidade a cada 400 $\mathrm{m}$ e da frequência cardíaca batimento a batimento. A média da PSE e da velocidade a cada quilômetro foi calculada e a estratégia de prova determinada pela curva da velocidade e distância.

\section{Risco de fadiga prematura}

Para a determinaçáo do risco de fadiga prematura foi utilizado procedimento adaptado ao proposto por De Koning et al. ${ }^{17}$, sendo utilizada a escala de BorG $^{22}$ de 15 pontos. A PSE foi coletada a cada 400 $\mathrm{m}$ e a média a cada quilômetro determinada. Para o cálculo do risco de fadiga prematura foi utilizado o produto da PSE momentânea pela distância restante de prova a cada quilômetro.

\section{Análise estatística}

Os resultados são apresentados como média \pm desvio padrão (DP). As diferenças na velocidade, PSE e risco de fadiga a cada quilômetro foram determinadas por meio de análise de variância (ANOVA) de um fator (distância) para medidas repetidas. A velocidade a cada quilômetro foi comparada com a média de velocidade da prova por meio da ANOVA de um fator. Quando um valor de F significante foi obtido o "post-hoc" com ajustamento de Tukey foi utilizado.

Foram calculadas as correlaçóes (coeficiente de correlação de Pearson) entre a PSE e a velocidade e entre o risco de fadiga com a velocidade a cada quilômetro. Para determinar a magnitude das correlaçóes foi utilizada a classificação proposta por $\operatorname{CoHEN}^{24}$. $\mathrm{O}$ nível de significância adotado foi de $\mathrm{p}<0,05$. 


\section{Resultados}

As características dos sujeitos podem ser observadas na TABELA 1.

TABELA 1 - Características dos sujeitos.

\begin{tabular}{lc}
\hline Variáveis & Média \pm DP \\
\hline Idade (anos) & $34,4 \pm 7,8$ \\
Massa corporal $(\mathrm{kg})$ & $69,2 \pm 7,8$ \\
Estatura $(\mathrm{cm})$ & $172,7 \pm 7,7$ \\
$\mathrm{VO}_{2}$ máx $(\mathrm{ml} / \mathrm{kg} / \mathrm{min})$ & $54,9 \pm 5,4$ \\
Tempo nos $10 \mathrm{~km}(\mathrm{~min}: \mathrm{s})$ & $41: 39 \pm 3: 52$ \\
\hline
\end{tabular}

A FIGURA 1A apresenta a velocidade de acordo com a distância, o que permitiu determinar a estratégia de prova utilizada. A velocidade média durante os $10 \mathrm{~km}$ foi $14,6 \pm 1,3 \mathrm{~km} \cdot \mathrm{h}^{-1}$. Os corredores percorreram o primeiro quilômetro de prova com velocidade $-8,1 \%$ mais alta do que a média $\left(V m 1 \mathrm{~km}=15,8 \pm 1,4 \mathrm{~km} \cdot \mathrm{h}^{-1} ; \mathrm{p} \leq 0,001\right)$. A $V m 1 \mathrm{~km}$ foi significantemente maior do que as velocidades no restante da prova $(\mathrm{p}<0,001)$. No segundo quilômetro a velocidade foi $-4,1 \%$ mais alta $\left(\operatorname{Vm} 2 \mathrm{~km}=15,2 \pm 1,3 \mathrm{~km} \cdot \mathrm{h}^{-1} ; \mathrm{p}=0,001\right)$ que a média da prova. A velocidade média por quilômetro diminuiu gradualmente ao longo da prova atingido valores menores do que a velocidade média nos quilômetros seis $\left(14,2 \pm 1,3 \mathrm{~km} \cdot \mathrm{h}^{-1} ; \mathrm{p}=0,04\right)$ sete $\left(14,2 \pm 1,3 \mathrm{~km} \cdot \mathrm{h}^{-1} ; \mathrm{p}=0,02\right)$ oito $\left(14,1 \pm 1,3 \mathrm{~km} \cdot \mathrm{h}^{-1}\right.$; $\mathrm{p}=0,01)$ e nove $\left(14,1 \pm 1,4 \mathrm{~km} \cdot \mathrm{h}^{-1} ; \mathrm{p}=0,01\right)$. No último quilômetro a velocidade aumentou novamente, caracterizando o "sprint"-final $(\mathrm{Vm} 10 \mathrm{~km}$ = $\left.14,7 \pm 1,6 \mathrm{~km} \cdot \mathrm{h}^{-1}\right)$.

O comportamento da PSE ao longo dos $10 \mathrm{~km}$ pode ser observado na FIGURA 1B. A PSE aumentou linearmente ao longo da prova. A PSE do primeiro quilômetro náo foi diferente do segundo quilômetro, no entanto, foi significantemente menor em relação ao restante da prova (terceiro ao décimo quilômetro; $\mathrm{p}=0,001)$. Do terceiro quilômetro em diante a PSE aumentou significantemente $(\mathrm{p}<$ $0,004)$ a cada quilômetro, chegando aos maiores valores no final dos $10 \mathrm{~km}(17 \pm 2)$.

Na FIGURA 1C podemos observar o comportamento do risco de fadiga prematura ao longo da corrida de $10 \mathrm{~km}$. O risco de fadiga diminuiu significantemente após o terceiro quilômetro $(\mathrm{p}<0,001)$ à medida que a distância restante foi sendo reduzida.

Foi detectada uma forte correlação negativa entre a PSE e a velocidade desenvolvida durante a prova $(\mathrm{r}=-0,80 ; \mathrm{p}=0,006$ FIGURA 2A). Por outro lado, apesar de significante, foi observada uma correlação moderada negativa entre o risco de fadiga e a velocidade $(\mathrm{r}=-0,57 ; \mathrm{p}=0,04$ FIGURA 2B).
As figuras representam os valores médios da amostra durante a corrida de $10 \mathrm{~km}$. Para a melhor visualização dos dados os desviospadrão foram retirados da figura.

Figura A: Estratégia de prova (velocidade $x$ distância);

Figura B: Percepção subjetiva de esforço (PSE);

Figura C: Risco de fadiga prematura.

${ }^{*} p \leq 0,001$ vs velocidade média de prova; $\& p \leq 0,004$ vs velocidade média de prova; $\# p \leq 0,001$ vs restante de prova;

$\$ p \leq 0,001$ vs quilômetro seguinte.

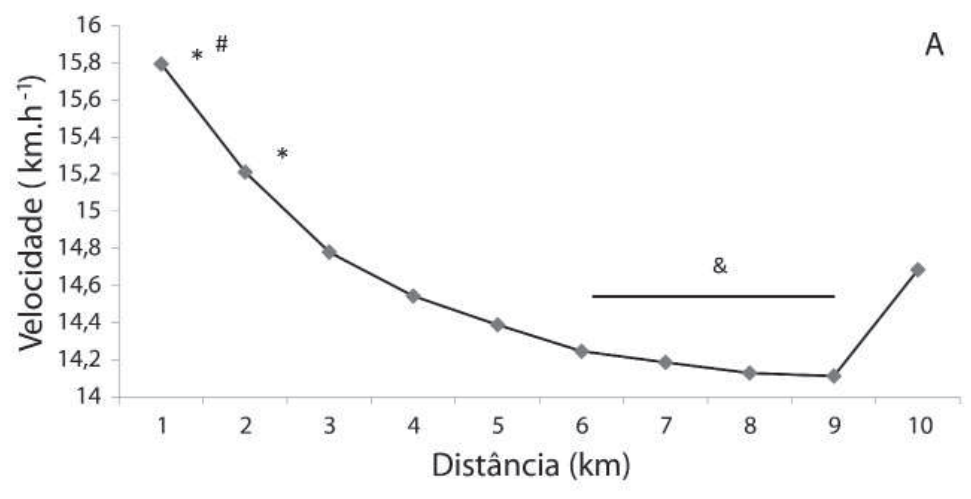

FIGURA 1 - Estratégia de prova (velocidade x distância), percepção subjetiva de esforço e risco de fadiga prematura durante a prova de $10 \mathrm{~km}$ (continua). 


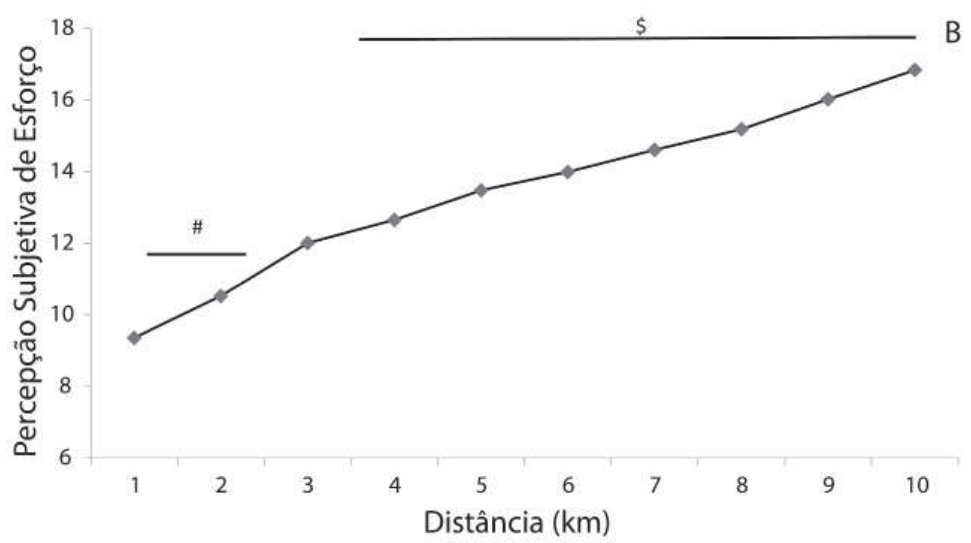

As figuras representam os valores médios da amostra durante a corrida de $10 \mathrm{~km}$. Para a melhor visualização dos dados os desviospadrão foram retirados da figura.

Figura C: Risco de fadiga prematura.

${ }^{*} p \leq 0,001$ vs velocidade média de prova;

$\& p \leq 0,004$ vs velocidade média de prova;

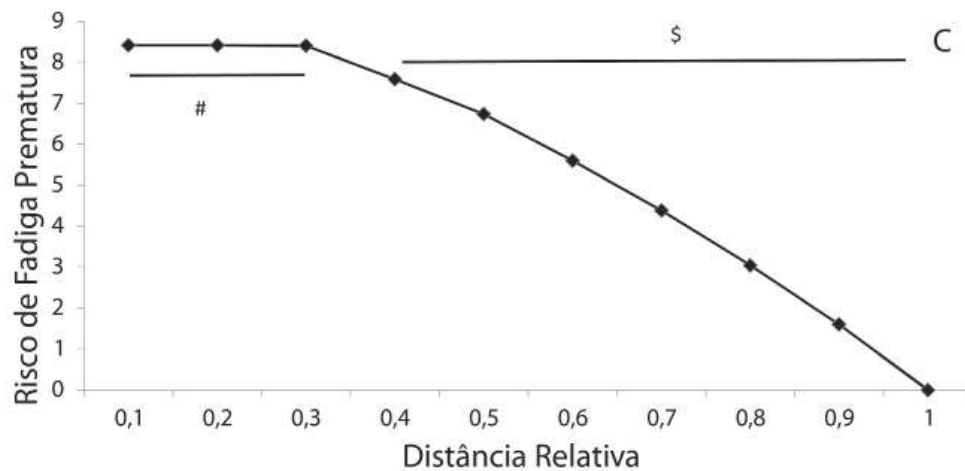

$\# p \leq 0,001$ vs restante de prova;

$\$ p \leq 0,001$ vs quilômetro seguinte.

Figura A: Correlação entre a PSE e a velocidade $\left(\mathrm{km} \cdot \mathrm{h}^{-1}\right)$ a cada quilômetro durante a prova de $10 \mathrm{~km}$; Figura B: Correlação entre o risco de fadiga e a velocidade $\left(\mathrm{km} \cdot \mathrm{h}^{-1}\right)$ a cada quilômetro durante a prova de $10 \mathrm{~km}$.

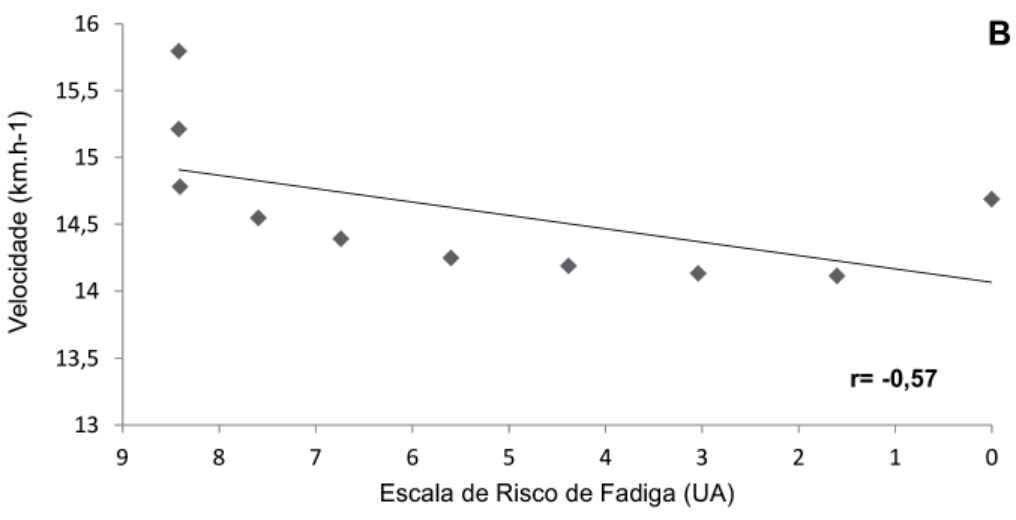

FIGURA 2 - Correlação entre a PSE e o risco de fadiga com a estratégia de prova durante a corrida de $10 \mathrm{~km}$.

Rev Bras Educ Fís Esporte, (São Paulo) 2015 Abr-Jun; 29(2):197-205 • 201 


\section{Discussão}

O objetivo do presente estudo foi verificar as modificaçóes induzidas sobre a estratégia de prova frente alteraçóes do risco de fadiga prematura e da PSE em corredores amadores durante uma corrida simulada de $10 \mathrm{~km}$ em pista. Nossos resultados mostraram uma forte correlação negativa entre a PSE e a velocidade durante a corrida $(r=-0,80$; FIGURA 2A). Assim, conforme a sensação de esforço foi aumentando ao longo da prova o atleta ajustou a sua velocidade, o que deve ter ocorrido para evitar uma possível fadiga prematura ${ }^{16}$. Nossos dados sugerem o importante papel da PSE sobre os ajustes de velocidade e consequentemente, sobre a estratégia de prova desenvolvida durante uma corrida de $10 \mathrm{~km}$.

Adicionalmente, próximo ao final da prova os corredores voltaram a aumentar a velocidade. Esse aumento foi observado apenas quando o risco de fadiga prematura apresentou valores baixos, menores do que dois. O papel do risco de fadiga prematura sobre a estratégia de prova pode ser sugerido na FIGURA $2 \mathrm{~A}$, na qual observamos a quebra da linearidade entre a velocidade e a PSE próximo ao final da prova, ocorrendo o aumento da velocidade no último quilômetro da corrida, mesmo com altos valores de PSE. A quebra da linearidade entre a PSE e a velocidade parece ter sido influenciada pela distância restante de prova e os baixos níveis de risco de fadiga prematura. De fato, nossos resultados corroboram com os observados por De Koning et al. ${ }^{17}$, onde a velocidade desenvolvida pelos atletas durante a prova aumentou quando o risco de fadiga prematura atingiu valores baixos, no caso, menores do que 1,5. Assim, com a proximidade do final da prova e o baixo risco de fadiga prematura, o atleta parece aumentar a velocidade sabendo que será capaz de percorrer a distância restante da prova ${ }^{17}$. Com isso, podemos supor que o risco de fadiga prematura parece ser um importante fator para a realização do "sprint"-final em uma prova de $10 \mathrm{~km}$ em pista.

No presente estudo, a estratégia de prova com início rápido em formato de "J-invertido" (FIGURA 1A) foi utilizada pelos corredores ${ }^{5}$, sendo os dois primeiros quilômetros realizados com velocidades mais altas do que a velocidade média de prova. A velocidade foi reduzida ao longo da prova, atingindo os valores mais baixos a partir do sexto quilômetro. Um novo aumento da velocidade foi observado no último quilômetro, caracterizando o "sprint"-final. De fato, as estratégias de padrão variável com início rápido têm sido utilizadas por muitos corredores em provas de médias e longas distâncias ${ }^{4,25}$. TuCKer et al. ${ }^{4}$ observaram esse tipo de estratégia em 33 dos 34 recordistas mundiais dos $10 \mathrm{~km}$. Lima-SiLva et al. ${ }^{3}$ observaram que corredores com tempo nos 10 $\mathrm{km}$ inferior a 35,6 min utilizavam a estratégia com início rápido com padrão em "U". Sendo assim, observamos que os corredores amadores no presente estudo, porém experientes em provas de $10 \mathrm{~km}$, ajustam a sua velocidade durante uma corrida com padróes semelhantes a atletas de alto desempenho.

A estratégia com início rápido parece ter ação direta sobre alguns mecanismos fisiológicos como o aumento na cinética do consumo de oxigênio $\left(\mathrm{VO}_{2}\right)$ e a maior eficiência na ressíntese de ATP ${ }^{26-}$ ${ }^{27}$. Para que altas velocidades sejam desenvolvidas, um maior número de fibras musculares e maior proporção de fibras do tipo II devem ser ativadas, o que levará a maior utilização de ATP no início do exercício, alterando a razão ATP/ADP. Essa alteração, associada à maior utilização do sistema ATP/CP, seriam responsáveis pela aceleração da cinética "on" do consumo de oxigênio, resultando na redução do déficit de oxigênio durante a prova. Isso permitiria ao atleta atingir o "steady state" mais rápido, gerando um menor estresse metabólico ${ }^{27-28}$.

Por outro lado, se as velocidades iniciais forem muito altas pode ocorrer uma queda de desempenho ${ }^{29}$. As altas velocidades podem induzir um grande desequilíbrio metabólico no início do exercício, o que prejudicaria o atleta durante o restante da prova, podendo induzir um quadro de fadiga prematura, impossibilitando a manutenção da velocidade e prejudicando o desempenho $\mathrm{o}^{7,17}$. Sendo assim, os ajustes de velocidade durante uma corrida devem ser realizados para prevenir grandes distúrbios fisiológicos e metabólicos, evitando um quadro de fadiga precoce ${ }^{16-17}$.

Um ponto importante, porém ainda bastante controverso, são os mecanismos pelos quais os corredores ajustam a velocidade ao longo da corrida. O modelo para explicar esses ajustes foi primeiramente proposto por $\mathrm{ULMER}^{30}$, sendo posteriormente ajustado e aplicado em provas de média e longa distâncias ${ }^{13-14,31}$. Nesse modelo, os ajustes sobre a intensidade do esforço são influenciados por fatores fisiológicos e psicológicos, onde estes são comparados momento a momento durante a prova e permitem que o atleta termine no menor tempo possível, sem entrar em fadiga prematura. Nesse sentido, a escala de PSE parece ter grande aplicabilidade no estudo da estratégia de prova, uma 
vez que ela parece refletir as respostas integradas de diferentes sistemas, combinando as alteraçóes fisiológicas e psicológicas ${ }^{8,15,17,32-33}$. De fato, os dados observados no presente estudo corroboram outros trabalhos que sugerem a importante influência da PSE sobre os ajustes da estratégia de prova ${ }^{15,17,34-35}$.

As altas velocidades iniciais observadas no nosso estudo podem ter ocorrido devido à baixa PSE; ou seja, os atletas se sentiam confortáveis para correr em altas velocidades. Esses resultados são suportados pelo estudo de BERTUZzI et al..$^{34}$. Os autores observaram que a PSE foi o único fator determinante da velocidade nos primeiros $400 \mathrm{~m}$ em uma corrida de $10 \mathrm{~km}$. Conforme a PSE foi aumentando durante a prova a velocidade de corrida foi diminuindo. A forte correlação negativa entre a velocidade e a PSE $(r=-0,80)$ parece ser quebrada apenas no último quilômetro, quando a velocidade aumenta novamente, mesmo com uma alta PSE. De fato, se o último quilômetro for retirado da análise, a correlação observada entre a PSE e a velocidade é ainda maior $(r=-0,96)$.

Em resumo, os achados do presente estudo confirmam os resultados observados na literatura ${ }^{15,17,34-35}$, sugerindo que corredores com nível amador, porém experientes em provas de média e longa distâncias, ajustam a estratégia de prova durante uma corrida de $10 \mathrm{~km}$ conforme a PSE. Assim como demonstrado por De Koning et al. ${ }^{17}$ em esteira, os resultados do presente estudo sugerem que em provas de pista o risco de fadiga prematura parece ser fundamental para a realização do "sprint"-final. Futuros estudos deveriam ser conduzidos no intuito de compreender melhor a influência a participação do risco de fadiga prematura durante um evento esportivo de média e longa duração e se esse poderia ser de alguma forma modulado, possibilitando um maior desempenho.

\begin{abstract}
Hazard score, rating of perceived exertion and pacing strategy during a $10 \mathrm{~km}$ running

The aim of the study was to verify the modifications on pacing strategy induced by the hazard score of premature fatigue and rated perceived exertion (RPE) in amateur runners during a simulated 10-km running. Fifty five amateur runners with the 10-km run time of 41:39 \pm 3:52 min:s participated in the study. The pacing strategy and the RPE were analyzed each kilometer. The hazard score of premature fatigue was expressed as the product of RPE by the remaining distance of the running. An ANOVA one-way for repeated measures was applied to determine possible statistical differences in speed, RPE and hazard score at each kilometer or the running speed differences at each kilometer and the average running speed. Pearson's product moment correlations were calculated between RPE and hazard of premature fatigue and running speed. The speed in the first kilometer was $8.1 \%$ higher than the average speed $(p \leq 0.001)$. There was a progressive decrease in speed during the running with an increment near the last kilometer. The RPE increased linearly until the end of the running and the hazard score of premature fatigue significantly decreased after the third kilometer. During the running there was a strong negative correlation between speed and RPE ( $r=-0.80 ; p=0.006)$. It was also observed a moderate negative correlation between speed and hazard score of premature fatigue $(r=-0.57 ; p=0.04)$. Thus, our results suggested the important role of RPE on speed adjustment during a 10-km running and the increase of running speed near the end of the race seems to be associated to the lower score of hazard of premature fatigue.
\end{abstract}

KEY WORDS: Athletic performance; Energy expanditure; Exercise tolerance; Fatigue. 


\section{Referências}

1. Foster C, DeKoning JJ, Hettinga F, et al. Effect of competitive distance on energy expenditure during simulated competition. Int J Sports Med. 2004;25:198-204.

2. Abbiss CR, Laursen PB. Describing and understanding pacing strategies during athletic competition. Sports Med. 2008;38:239-252.

3. Lima-Silva AE, Bertuzzi RC, Pires FO, et al. Effect of performance level on pacing strategy during a 10-km running race. Eur J Appl Physiol. 2010;108:1045-53.

4. Tucker R, Lambert MI, Noakes TD. An analysis of pacing strategies during men's world-record performances in track athletics. Int J Sport Physiol. 2006;1:233-45.

5. Carmo EC, Barreti DLM, Ugrinowitsch C, Tricoli V. Estratégia de corrida em média e longa distância: como ocorrem os ajustes de velocidade ao longo da prova? Rev Bras Educ Fís Esporte. 2012;26:351-63.

6. Atkinson G, Peacock O, Law M. Acceptability of power variation during a simulated hilly time trial. Int J Sports Med. 2007;28:157-63.

7. Gosztyla AE, Edwards DG, Quinn TJ, Kenefick RW. The impact of different pacing strategies on five-kilometer running time trial performance. J Strength Cond Res. 2006;20:882-6.

8. St Clair Gibson A, Baden DA, Lambert MI, et al. The conscious perception of the sensation of fatigue. Sports Med. 2003;33:167-76.

9. Noakes TD, St Clair Gibson A. Logical limitations to the "catastrophe" models of fatigue during exercise in humans. Brit J Sport Med. 2004;38:648-9.

10. Noakes TD, St Clair Gibson A, Lambert EV. From catastrophe to complexity: a novel model of integrative central neural regulation of effort and fatigue during exercise in humans. Brit J Sport Med. 2004;38:511-4.

11. Baron B, Moullan F, Deruelle F, Noakes TD. The role of emotions on pacing strategies and performance in middle and long duration sport events. Brit J Sport Med. 2011;45:511-7.

12. Noakes TD, St Clair Gibson A, Lambert EV. From catastrophe to complexity: a novel model of integrative central neural regulation of effort and fatigue during exercise in humans: summary and conclusions. Brit J Sport Med. 2005;39:120-4.

13. Noakes TD. The central governor model of exercise regulation applied to the marathon. Sports Med. 2007;37: 374-7.

14. Tucker R, Noakes TD. The physiological regulation of pacing strategy during exercise: a critical review. Brit J Sport Med. 2009;43:e1.

15. Joseph T, Johnson B, Battista RA, et al. Perception of fatigue during simulated competition. Med Sci Sport Exerc. 2008;40:381-6.

16. Tucker R. The anticipatory regulation of performance: the physiological basis for pacing strategies and the development of a perception-based model for exercise performance. Brit J Sport Med. 2009;43:392-400.

17. de Koning JJ, Foster C, Bakkum A, et al. Regulation of pacing strategy during athletic competition. Plos One. 2011;20:6.

18. Marcora SM. Perception of effort during exercise is independent of afferent feedback from skeletal muscles, heart, and lungs. J Appl Physiol. 2009;106:2060-2.

19. Marcora SM, Bosio A, de Morree HM. Locomotor muscle fatigue increases cardiorespiratory responses and reduces performance during intense cycling exercise independently from metabolic stress. Am J Physiol Regul Integr Comp Physiol. 2008;294:R874-883.

20. Renfree A, Martin L, Richards A, St Clair Gibson A. All for one and one for all! Disparity between overall crew's and individual rowers' pacing strategies during rowing. Int J Sports Physiol Perform. 2012;7:298-300.

21. Fernandes RJ, Billat VL, Cruz AC, Colaco PJ, Cardoso CS, Vilas-Boas JP. Does net energy cost of swimming affect time to exhaustion at the individual's maximal oxygen consumption velocity? J Sport Med Phys Fit. 2006;46:373-80.

22. Borg GAV. Ratings of perceived exertion and heart-rates during short-term cycle exercise and their use in a new cycling strength test. Int J Sports Med. 1982;3:153-8.

23. Hardy CJ, Rejeski WJ. Not what, but how one feels: the measurement of affect during exercise. J Sport Exerc Psychol. 1989;11:304-17.

24. Cohen J. Statistical power analysis for the behavioral sciences. 2nd ed. Hillsdale: L. Erlbaum; 1988.

25. Bertuzzi R, Nakamura FY, Rossi LC, Kiss MA, Franchini E. Independência temporal das respostas do esforço percebido e da frequência cardíaca em relação à velocidade de corrida na simulação de uma prova de $10 \mathrm{~km}$. Rev Bras Med Esporte. 2006;12:179-83.

26. Foster C, De Koning JJ, Hettinga F, et al. Pattern of energy expenditure during simulated competition. Med Sci Sport Exerc. 2003;35:826-31. 
27. Bishop D, Bonetti D, Dawson B. The influence of pacing strategy on $\mathrm{VO}_{2}$ and supramaximal kayak performance. Med Sci Sport Exerc. 2002;34:1041-7.

28. Hanon C, Leveque JM, Thomas C, Vivier L. Pacing strategy and $\mathrm{VO}_{2}$ kinetics during a $1500-\mathrm{m}$ race. Int J Sports Med. 2008;29:206-11.

29. Carmo EC, Gil S, Kobal R, et al. Fast start strategy induces different individual responses in velocity regulation during a $10 \mathrm{~km}$ race. 17 Annual Congress of the European College of Sport Science; 2012; July 4-7; Bruges, BEL. Bruges: European College of Sport Science; 2002. p.112. Available from: http://www.ed.ac.uk/polopoly_fs/1.94449!/fileManager/Book\%20of\%20Abstracts\%20ECSS\%20Bruges\%202012.pdf.

30. Ulmer HV. Concept of an extracellular regulation of muscular metabolic rate during heavy exercise in humans by psychophysiological feedback. Experientia. 1996;52:416-20.

31. Rauch HG, St Clair Gibson A, Lambert EV, Noakes TD. A signalling role for muscle glycogen in the regulation of pace during prolonged exercise. Brit J Sport Med. 2005;39:34-8.

32. Faulkner J, Arnold T, Eston R. Effect of accurate and inaccurate distance feedback on performance markers and pacing strategies during running. Scand J Med Sci Sport. 2011;21:E176-83.

33. Faulkner J, Parfitt G, Eston R. The rating of perceived exertion during competitive running scales with time. Psychophysiology. 2008;45:977-85.

34. Bertuzzi R, Lima-Silva AE, Pires FO, et al. Pacing strategy determinants during a 10-km running time trial: contributions of perceived effort, physiological, and muscular parameters. J Strength Cond Res. 2014;28:1688-96.

35. Noakes TD. Linear relationship between the perception of effort and the duration of constant load exercise that remains. J Appl Physiol. 2004;96:1571-2.

\section{Agradecimentos}

A FAPESP (processo 08-50934-1) e ao CNPq (processos 304814/2010-5 e 474105/2011-3) pelo apoio financeiro.

\begin{tabular}{|c|c|}
\hline $\begin{array}{r}\text { ENDEREÇO } \\
\text { Everton Crivoi do Carmo } \\
\text { Escola de Educação Física e Esporte - USP } \\
\text { Av. Prof. Mello Moraes, } 65 \\
\text { 05508-030 - São Paulo - SP - BRASIL } \\
\text { e-mail: evertoncrivoi@usp.br }\end{array}$ & $\begin{array}{l}\text { Recebido para publicação: 18/03/2014 } \\
\text { Revisado: 22/12/2014 } \\
\text { Aceito: 27/04/2015 }\end{array}$ \\
\hline
\end{tabular}

